\title{
Für eine neue Kultur der Mobilität
}

Für die Verkehrswende brauchen wir neue Wege nachhaltiger Mobilität: alternative Antriebe und Kraftstoffe, effiziente und vernetzte Technologien, aber auch klare politische Leitbilder, angepasste Instrumente und Bewegung im Habitus, also eine neue Kultur der Mobilität.

Von Winfried Hermann

W ir wollen mehr Mobilität und Lebensqualität, nicht mehr Verkehr. Die Anforderungen sind groß: Der Klimawandel erfordert die Abkehr von der fossil getriebenen Mobilität, die Digitalisierung verändert das Auto und wie wir unterwegs sind. Fakt ist auch: Das derzeitige Verkehrssystem ist längst schon an seine Grenzen gelangt. Im (Dauer-)Stau aus Individualverkehr und anhaltender Güterverlagerung auf die Straße blockiert sich der (Auto-)Verkehr selbst. Nicht nur entlang der großen Achsen leiden Anwohner in Städten und Gemeinden unter Lärm, Luftverschmutzung und Stress.

Der Verkehr ist weltweit ein Klimaschutzproblem. Mit dem Pariser Klimaschutzabkommen sind wir in einer völkerrechtlich verbindlichen Pflicht, hierauf zu reagieren. Heute ist klar: Alle bisher vorliegenden nationalen Klimaziele würden die Erderwärmung lediglich auf drei Grad begrenzen. Um das 1,5-Grad-Ziel zu erreichen, müssten weltweite Emissionen jedoch ab sofort jährlich um $5 \%$ sinken. Im Verkehrssektor, dem Stiefkind der deutschen Klimaschutzpolitik, verlangt der Pariser Beschluss radikale Veränderungen, denn sein Anteil an den Treibhausgas-Emissionen nimmt zu und nicht ab (1990: 13\%, 2015: 18\%). In Baden-Württemberg ist der Verkehrssektor der größte $\mathrm{CO}_{2}$-Emittent und verantwortlich für ein Drittel der Treibhausgase.

\section{Transformationszwang für das industrie- politische Überleben}

Es ist kein Geheimnis, dass Wohlstand und Ansehen in Baden-Württemberg und Deutschland an Produktion und Verkauf von hochwertigen Fahrzeugen hängen. Tausende Arbeitsplätze bei Herstellern und Zulieferern sind damit verbunden. Für die Landesregierung ist es auch deshalb ein Kernanliegen, Baden-Württembergs Automobilwirtschaft erfolgreich in die Zukunft zu führen. Schon im Mai 2017 hat die Landesregie- rung mit der heimischen Automobilbranche, Verbänden, Gewerkschaften und Wissenschaft einen Strategiedialog über den Transformationsprozess in der Automobilwirtschaft gestartet.

Die Branche steht vor einem tief greifenden Umbruch. Wer morgen noch Fahrzeuge exportieren will, muss sich heute bewegen und wer hier auf die Bremse tritt, schadet der Wirtschaft und den Arbeitsplätzen! Deshalb brauchen wir einen klaren Rahmen für die Transformation zur emissionsfreien Mobilität. Bei der „Transformationsagenda“ stehen Forschung, Entwicklung und Realisierung alternativer Antriebe und Kraftstoffe, Digitalisierung, Elektromobilität und Innovationen für die Mobilität der Zukunft im Fokus.

Aber Technik allein wird die Probleme nicht lösen. Denn nicht alles, was technisch möglich ist, ist auch zukunftsfähig. Unser Verkehrssystem muss zu einem Mobilitätssystem entwickelt werden, in dem das Auto einen anderen Stellenwert einnimmt und seine Dominanz verliert. Die neuen Treiber Digitalisierung und Vernetzung verändern den Verkehr in seinen Grundzügen. Das Auto wird autonomer und das Verkehrssystem zunehmend digital und vernetzt. Das Angebot an Mobilitätsdienstleistungen für multimodales Verkehrsverhalten wächst rasant. Dabei beeinflusst Digitalisierung schon heute, welche Verkehrsträger genutzt und welche Wege gefahren werden. Das Smartphone wird dabei zum Mobilitätsnavigator. Dazu brauchen wir dringend Wirkungsforschung, denn die Folgen der schönen neuen Smartphonewelt für Datenschutz oder das Verkehrsaufkommen müssen untersucht und sinnvoll geregelt werden. Es ist heute nicht ausgemacht, ob die multimodal mobilen jungen Leute Rad fahren oder in den öffentlichen Verkehr umsteigen oder ob sie sich vermehrt vom digital gesteuerten Auto fahren lassen, während sie in sozialen Medien virtuell unterwegs sind.

Es ist daher enorm wichtig, die neuen Technologiechancen auch für den öffentlichen Personennahverkehr (ÖPNV) nutzbar zu machen. Simulationen aus Lissabon, Pittsburg und Singapur zeigen: Mit einer effizienzorientierten Automatisierung und Digitalisierung wären für motorisierten Individualverkehr (MIV) und ÖPNV nur noch 10-30\% des Fahrzeugbestands nötig, Kommunen hätten so weniger Park- und Verkehrsflächen, Anwohner/innen mehr Lebensqualität und -raum. Den Menschen ist genau das auch zunehmend wichtiger und im veränderten Mobilitätsverhalten wird Nutzen wichtiger als Besitzen.

Für die Zukunft der Mobilität ist weniger mehr! Im Verkehrsministerium Baden-Württemberg entwickeln wir verschiedene Ansätze, um die Chancen des autonomen Verkehrs (deutlich weniger Fahrzeuge) mit der Strategie integrierter Mo- 
bilitätskonzepte zu nutzen. Wir setzen damit den Weg der ökologischen Modernisierung fort, den das Land mit der Regierungsübernahme 2011 begonnen hat und wollen zum Wegbereiter einer modernen und nachhaltigen Mobilität der Zukunft werden. Für Politik und Verwaltung gilt es, die Megatrends der Mobilität aufzugreifen und in eine ökologisch, wirtschaftlich und sozial verträgliche Richtung zu lenken.

Aus globaler Sicht hat die Verkehrswende längst begonnen, weltweit werden neue Wege gesucht und bestritten, Menschen und Güter von A nach B zu bewegen. Norwegen ist zum Beispiel Vorreiter bei der E-Mobilität, die Schweiz und Österreich bauen moderne Bahn- und ÖPNV-Netze, in Kopenhagen und Amsterdam hat der Radverkehr Vorfahrt. Baden-Württemberg als Land der Tüftler hat beste Voraussetzung, um Technologieführer bei intelligenten, ressourcen- und klimaschonenden Technologien für die Mobilität von morgen zu sein.

Es geht um nicht weniger als die Neuerfindung des Autos und der Mobilität! Viele haben den Beschluss der Grünen zum Ausstieg aus dem fossilen Verbrennungsmotor kritisiert. Aus meiner Sicht kann man über ein genaues Datum dafür streiten, bedeutsam dabei ist die Botschaft: rechtzeitig umsteigen! Denn wir brauchen eine realistische und ambitionierte Transformationsstrategie auch und vor allem, weil wir als Politik auch Verantwortung für Arbeitsplätze tragen.

\section{Strategie für moderne und nachhaltige Mobilität}

Den Weg hin zu einer modernen und nachhaltigen Mobilität umschreibt mein Ministerium mit der „Strategie der fünf V“: Wir möchten die Verkehrsmittel ökologischer gestalten und Verkehrsinfrastruktur verbessern. Wir wollen Verkehr auf ökologisch sinnvolle Verkehrsträger verlagern. Wir möchten Verkehrsströme intelligent vernetzen. Wir wollen überflüssigen motorisierten Verkehr vermeiden und selber Vorbild und Vorreiter sein und dadurch für eine neue Mobilität werben.

Was tun wir konkret? Mit der dritten Landesinitiative Elektromobilität werden wir bis 2021 circa 40 Millionen Euro in Ladeinfrastruktur, ausgewählte Fahrzeugflotten und innovative Vorhaben investieren.

Das Rückgrat des Umweltverbundes ist der ÖPNV mit Bus und Bahn. Mit der Zukunftsoffensive für Bahnen und Busse wollen wir die Fahrgastzahlen bis 2030 deutlich erhöhen. Dazu gehört ein bis 2025 landesweit verlässliches Grundangebot mit Bahn, Bus und Rufbussen im Stundentakt von frühmorgens bis spätabends. Mit neuen Nahverkehrszügen im Landesdesign setzen wir Qualitätsmaßstäbe für einen attraktiven Schienenpersonennahverkehr (SPNV), barrierefrei, funktional und vernetzt.

Da $50 \%$ der Pkw-Fahrten in Städten kürzer als fünf Kilometer sind, können wir einen Teil der Pkw-Fahrten auf Fußund Radverkehr verlagern und emissionsfrei zurücklegen. Wir wollen das Land im Alltags- und Freizeitverkehr noch fahrradfreundlicher machen und mittelfristig den Anteil des Radver- kehrs auf 20\% verdoppeln. Dazu entwickelten wir in einem breiten Beteiligungsprozess unsere RadSTRATEGIE 2015. Dafür haben wir Mittel für Radwege an Landesstraßen aufgestockt, ein Sonderprogramm Radschnellwege aufgelegt, ein Erhaltungsmanagement für Radwege eingeführt und die Fahrradmitnahme im ÖPNV verbessert.

$23 \%$ aller Wege werden zu Fuß zurückgelegt, als erstes Flächenland entwickeln wir eine systematische Fußverkehrsförderung und wollen bis 2030 den Fußverkehrsanteil auf 30\% steigern. Ein hoher Fußverkehrsanteil ist Indikator für hohe Lebensqualität. Wir unterstützen hierfür unsere Kommunen.

Insbesondere mit Blick auf den Güterverkehr wird deutlich, dass der Bundesverkehrswegeplan $2030 \mathrm{zu}$ wenig für die Schiene enthält und viele Projekte noch nicht einmal bewertet sind. Für uns stehen wichtige Einzelprojekte wie die Strecke Stuttgart - Zürich und der Ausbau der Rheintalbahn auf einer bürger- und umweltfreundlichen Trasse an. Die Verkehrsverlagerung erfordert den Ausbau des Schienennetzes und der Verladeanlagen für den kombinierten Verkehr.

Verkehr verursacht 20-25\% des Flächenverbrauchs. Mit einer verbesserten Innenentwicklung schaffen wir kompakte Siedlungsstrukturen und schaffen mit dem Förderprogramm Flächen gewinnen durch Innenentwicklung Vorrang für flächensparende Verkehrsmittel. Für die Verknüpfung von Wohn- und Arbeitswelt brauchen wir eine gute Nah- und Grundversorgung, das heißt den öffentlichen Verkehr (ÖV) schon bei der Siedlungsentwicklung zu berücksichtigen, etwa mit einem Mobilitäts- und Parkraumanagement und Fahrzeugverleihsystemen.

Das Land fördert gemeinwohlorientierte Ansätze in Verkehrssteuerung und neue bedarfsorientierte Mobilitätsangebote, insbesondere im ländlichen Raum. Als Vorreiter für nachhaltige Mobilität fördert das Land mit einer Beschaffungsinitiative die Umrüstung des Fuhrparks auf E-Fahrzeuge beziehungsweise Hybridfahrzeuge. Es ist viel Bewegung im Land. Aber natürlich wissen wir auch, dass noch $80 \%$ unseres Verkehrs auf der Straße passiert. Deswegen hat moderne Mobilitätspolitik alle Verkehrsträger/innen- und Teilnehmer/innen für die Mobilitätswende im Fokus. Dafür müssen wir die Technologie ändern, aber auch die Mobilitätsmuster. Für die Mobilität der Zukunft brauchen wir Mut und Entschlossenheit und eine kluge politische Strategie: Weg vom Verkehr, hin zu neuer Mobilität. Und zwar zu einer menschengerechten, klimaschonenden, ökologisch und sozial gerechten Mobilität. Diese Wende gilt es auch gegen Widerstände zu gestalten. Nachhaltige Mobilität beginnt im Kopf, sie sollte dort aber nicht enden.

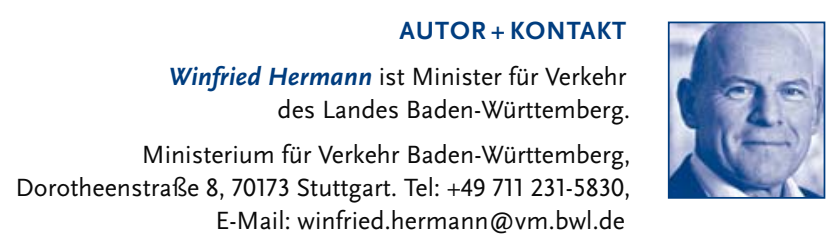

